\title{
The Use of Self-contained Range and Azimuth Measuring Apparatus to Detect Collision Courses
}

$$
\text { from J. S. Morrel }
$$

SOME readers of the January issue of the Journal may have noticed rather strongly discrepant statements on the subject of self-contained detection devices in the several papers on collision avoidance. For example, on page 43 Gaudillère 1 suggests either constant bearing or constant closing speed as an indicator of impending collision between aircraft; and on pages 82-87 Whitfield and Cade 2 suggest that ships may determine transverse velocity (and hence may identify a collision course) by using a differentiated doppler radar return. But on pages 25-26 the author of this note states ${ }^{3}$ that the derivatives of range and azimuth required for this use are in fact too small for successful physical measurement.

This is the crux of the matter. The geometry of the collision course is simple, elementary, and familiar. It is the same for ships at sea and for aircraft in flight (ignoring the third dimension). If the two craft involved are in unaccelerated motion and continue so, they will collide if, but only if, (I) the range $r$ is decreasing $(\dot{r}<0)$ and $(2)$ the relative azimuth $\theta$ is constant $(\dot{\theta}=0)$ or the relative closing speed is constant $(\ddot{r}=0)$. The second condition states only alternate choices of what to observe, not alternate physical situations; for under the hypothesis of unaccelerated motion the vanishing of either derivative implies the vanishing of the other also.

Mathematically, the phrase 'equals zero' is one end of a dichotomy, so to speak. Either a quantity vanishes or it doesn't. But alas, this is not true physically. Unless he gets his results solely by counting or by a process strictly equivalent to it (and sometimes not even then), the investigator of a physical situation never in fact has the privilege of saying 'equals zero'. He can only say his result is so small he can't say it isn't zero. If his means of observation are very good he can assign relatively tight bounds to the area of his uncertainty; if these means are poor the bounds will be correspondingly wide.

In the present case, therefore, it is not enough to look at the simple geometry. We must go further, in two directions, and ask (a) how big are $\dot{\theta}$ and $\vec{r}$ when the course is really not a collision course; (b) how big must they be before we can see they are not zero.

Their values depend on instant range $(r)$, future minimum range $(m)$, and relative speed $(v)$. The equations are

$$
\begin{aligned}
\dot{r}^{2} & =v^{2}\left(1-m^{2} r^{-2}\right) \\
\ddot{r} & =m^{2} V^{2} r^{-3} \\
-\dot{\theta} & =m v r^{-2}
\end{aligned}
$$

Equation (I) shows the difference between closing speed $\dot{r}$ and relative speed $v$. It reflects the fact that the former is constant and equal to the latter only in the 
collision case $m=0$ (or the physically meaningless case $r=\infty$ ); but that otherwise $\boldsymbol{i}$ is variable and always less than $v$. The facts we are after lie in Equations (2) and (3).

Consider two aircraft approaching one another with relative speed $v=300$ knots, and now two nautical miles apart. If they are due to collide at all, they will do so in 24 seconds-a dreadfully short time in which to discover impending destruction and avoid it. But suppose there really isn't any danger; if nothing is done at all, the two will clear by a thousand feet. In terms of feet and seconds, $v=(300 \times 6080) \div 3600, r=2 \times 6080, m=1000$, and

$$
r=(300)^{2} \times(1000)^{2} \div 8 \times 6080 \times(3600)^{2}=0.14 \mathrm{ft} / \mathrm{sec}^{2}
$$

This is a little over $1 / 250$ gravity. And this is not the worst. Ten seconds before, $\ddot{r}$ was only a third of this. Ten seconds later it will be about five times as great. But this will be too late. Moreover it will be parlously small even then. And the very rapidity of its change will make measurement difficult. If the measuring apparatus has a long time-constant, it will lose the benefit of the later and larger values by phase lag; but if the time-constant is short, it will be prey to noise.

If these figures are not enough, it is easy to examine the matter in terms of actual ranges. Make a table of actual ranges for the $1000-\mathrm{ft}$. miss case above, say for one-second intervals from 30 to 20 seconds. Make a second table for an aircraft on a collision course at the same average range and the same average closing rate. The two sets of ranges will not differ at any time by more than one foot. The finest radar could not possibly distinguish the one case from the other. One might also consider the implications as to doppler radar, in terms of the frequency shift which must be detected. Put in terms of the actual numbers, the results are equally discouraging.

The example chosen is by no means a critical one. The relative speed is only a third of the maximum which may occur between aircraft now operational. The postulated minimum range is just equal to the currently accepted standard vertical separation, no greater. And the figures quoted take no account of the fact that to measure requires far greater accuracy than merely to detect. Neither do they take account of the fact that the $\ddot{r}$ referred to is a purely geometrical effect due only to the reference system in which the motion is observed, and not to any physical force. But in fact small physical forces do continuallyact on all aircraft in flight, and do produce physical accelerations whose effects could not be separated from the geometrical ones by any remote observing device. For instance, a trim error causing either aircraft to fly at a bank angle of just 4 milliradians would suffice to produce physically a $1 / 250$-gravity value of $\dot{r}$, quite regardless of the situation as to collision. Thus it might totally conceal an actual collision course; or by cancelling out the geometrical effect it might cause an indication of collision when in fact no danger existed. And these effects might occur even if the radar or other range-measuring device were an ideally perfect one.

Such a physical $\ddot{r}$ as this ordinarily could not be detected even in the cockpit of the craft experiencing it. For ordinary operation a vertical reference, itself accurate to 4 milliradians, is neither needed nor available. The only telltale would be heading change; this would depend not on relative speed but on the airspeed of the craft itself. For a $250-\mathrm{knot}$ craft, say, it would be only about $\frac{1}{3}^{\circ}$ per minute. So far as the remote observer is concerned, a few seconds duration would be enough, for he has no more time to look. 
Moreover, physical accelerations of the size stated are the rule in flight, not the exception, even on autopilot, and in smooth air. They can result from so common and usual an occurrence as a stewardess walking down the aisle. The truth is that an aircraft and its pilot (or autopilot) flying a course or a heading constitute a closed-loop, null-seeking system, continually wandering in curves about the null. In good conditions these curves are long and slow, but they are there, and it is accelerations that produce them. To see how long and slow: the turn radius of the $250-\mathrm{knot}$ craft at the $\frac{1}{3}^{\circ}$ per-minute heading rate is 200 nautical miles and its maximum deviation from an r.m.s. linear path during one minute is considerably less than its own wing span. Truly unaccelerated flight, even for a few seconds, is probably comparable in rarity to thirteen trumps in hand at the bridge table.

It has sometimes been argued that one of the major collision hazards is the case of two craft cruising on slowly converging courses, and hence at low closing speed; and that an $\ddot{i}$ scheme would give protection in these cases, even if not effective in high-speed encounters. Not so. Consider two craft half-a-mile apart, closing at 60 knots, due to clear by $500 \mathrm{ft}$. (The C.A.B. hearing evidence shows that the closing speed in the Grand Canyon case, often cited as an example of the low speed encounter, was surely higher than this, and probably was twice as high.) Here $\ddot{r}=0.09 \mathrm{ft} / \mathrm{sec}^{2}<1 / 300$ gravity, less than in the previous example. But if collision were due to occur, it would be only 30 seconds away. The conclusion seems forced that for aircraft the $\ddot{i}$ technique, however implemented, is not capable of distinguishing danger from safety in any case, unless the actual miss is so great that there is no need of knowing.

But what of ships at sea? Worse yet. I believe a single simple example will prove this. Suppose two ships cruising at 10 knots each on reciprocal headings, now only half-a-mile apart, due to pass clear by $1000 \mathrm{ft}$. as they. go. The instantaneous closing speed is $32 \mathrm{ft} / \mathrm{sec}$, , and collision would occur if at all in one minute and 35 seconds. But

$$
\ddot{r}=\left(3^{2}\right)^{2} \times(1000)^{2} \div(3040)^{3}=0.036 \mathrm{ft} / \mathrm{sec}^{2}=1 / 900 \text { gravity }
$$

The corresponding physical acceleration would be about $\mathrm{I} \cdot 3$ knots per minute.

The cautious navigator might wish to have the facts at greater range, say one mile. He would have to ask for a doppler radar capable of measuring an acceleration equivalent to ten knots per hour. He would also have to ask for a combination of wind, sea and power-plant controls in both ships which would prohibit shortterm variations of this magnitude in the actual speed of either ship. The really timid soul who wanted to know at two miles would have to ask for one knot per hour. If he and his fellow were both timid souls; both relied on radars not this good, thus failing to detect the thousand feet; both took alarm and turned on Whitfield and Cade's one-mile radius, one to port, the other to starboardwell, the plotting board will quickly reveal the sequel, but only to the survivors.

Space prohibits extended discussion of the angular measurement case; but it is 'little more encouraging. Taking the same numerical cases as before:

(I) Aircraft closing at 300 knots, 2 miles apart, due to miss by $1000 \mathrm{ft}$. $-\dot{\theta}=3.4 \mathrm{mils} / \mathrm{sec}$. (At this range a large aircraft itself subtends about 9 mils.)

(2) Aircraft closing at 60 knots, $\frac{1}{2}$ mile apart, due to miss by $500 \mathrm{ft}$. $-\dot{\theta}=$ $5.6 \mathrm{mils} / \mathrm{sec}$. (At this range a large aircraft subtends 30 to $4^{\circ}$ mils.) 
(3) Ships closing at 20 knots, $\frac{1}{2}$ mile apart, due to miss by $1000 \mathrm{ft} .-\dot{\theta}=$ $3.5 \mathrm{mils} / \mathrm{sec}$. (At this range a small ship, bow to, subtends $15-20 \mathrm{mils}$.)

(4) Ships, as in (3) except 2 miles apart. $-\dot{\theta}=0.2 \mathrm{mils} / \mathrm{sec}$. (At this range a large ship, broadside to, might subtend as much as 50 mils.)

It is apparent from these figures that any angle-measuring device will be useless either in the air or at sea, unless it is well stabilized. This is possible. But it is not cheap or easy, especially in the air, where weight and size are of paramount concern.

It is also apparent that the angular discrimination must be fine. This will demand a narrow beam, or its equivalent, which requires either large antennas or very high frequencies. The difficulties are serious in either case. And a narrow beam is not well adapted to discovery, i.e., to scanning large segments of space at a rapid rate.

One might go on to mention propagation anomalies, noise, power problems. Considering the subtended angles quoted above even 'glint' or scintillation of the target itself would sometimes be a source of serious error.

In the light of the facts and figures, the author of this note is convinced that all forms of radar or other remote observing devices (not including the human eye) can at best only play the role of elements in a system for collision avoidance, either in the air or at sea. They are inherently not capable of discovering collision risks if used alone. The information they supply is useful, but only if combined with other information secured by quite other means.

\section{REFEREN CES}

1 Gaudillère, P. (1958). Visual and electronic methods of preventing collision. This Journal, I 1, 40.

2 Whitfield, H. R. and Cade, C. M. (1958). A doppler collision course indicator for use at sea. This Journal, 11, 81 .

3 Morrel, J. S. (1958). The mathematics of collision avoidance in the air. This Journal, I I, 18 .

\section{M. Cade and H. R. Whitfield comment:}

Dr. Morrel's interesting note clearly delineates the difficulties of measuring small relative accelerations, particularly when spurious terms (e.g. due to yaw) are present. We do not, however, agree with Dr. Morrel's remarks concerning 'the really timid soul who wanted to know (the relative course and speed) at two miles . . .' Most mariners of our acquaintance want to know at three miles, or maybe even more.

As we explained (p. 86), our intention is that the doppler system should be used to examine targets for possible danger (either a steady bearing or a high approach velocity) and then to plot, since (p. 87) 'the doppler radar technique does not supersede plotting, nor is it intended to do so'. We consider (and so, we feel, will our friends the timid mariners) that "the ability rapidly to measure the approach velocity of several vessels in succession' (p. 87) is of value, even though they may all have indetectably small transverse components of velocity which would in fact indicate that they would clear own ship by a safe marginbarring any last-minute manœuvre. 